ARTICLE

Received 6 Feb 2014 | Accepted 1 Apr 2014 | Published 6 May 2014

DOl: $10.1038 /$ ncomms 4778

\title{
Enhanced flexibility of place discrimination learning by targeting striatal cholinergic interneurons
}

\author{
Kana Okada ${ }^{1}$, Kayo Nishizawa ${ }^{2}$, Ryoji Fukabori ${ }^{2}$, Nobuyuki Kai ${ }^{2}$, Akira Shiota $^{3}$, Masatsugu Ueda ${ }^{3}$, Yuji Tsutsui ${ }^{4}$, \\ Shogo Sakata', Natsuki Matsushita ${ }^{5} \&$ Kazuto Kobayashi ${ }^{2,6}$
}

Behavioural flexibility is mediated through the neural circuitry linking the prefrontal cortex and basal ganglia. Here we conduct selective elimination of striatal cholinergic interneurons in transgenic rats by immunotoxin-mediated cell targeting. Elimination of cholinergic interneurons from the dorsomedial striatum (DMS), but not from the dorsolateral striatum, results in enhanced reversal and extinction learning, sparing the acquisition of place discrimination. This enhancement is prevented by infusion of a non-selective muscarinic acetylcholine receptor agonist into the DMS either in the acquisition, reversal or extinction phase. In addition, gene-specific silencing of $\mathrm{M}_{4}$ muscarinic receptor by lentiviral expression of short hairpin RNA (shRNA) mimics the place reversal learning promoted by cholinergic elimination, whereas shRNA-mediated gene silencing of $M_{1}$ muscarinic receptor shows the normal performance of reversal learning. Our data indicate that DMS cholinergic interneurons inhibit behavioural flexibility, mainly through the $M_{4}$ muscarinic receptor, suggesting that this role is engaged to the stabilization of acquired reward contingency and the suppression of response switch to changed contingency.

\footnotetext{
${ }^{1}$ Department of Behavioural Sciences, Graduate School of Integrated Arts \& Sciences, Hiroshima University, Higashi-Hiroshima 739-8521, Japan.

${ }^{2}$ Department of Molecular Genetics, Institute of Biomedical Sciences, Fukushima Medical University School of Medicine, Fukushima 960-1295, Japan.

${ }^{3}$ PhoenixBio, Co., Ltd, Utsunomiya 321-0973, Japan. ${ }^{4}$ Faculty of Symbiotic Systems Science, Fukushima University, Fukushima 960-1296, Japan.

${ }^{5}$ Translational Research Center, Ehime University Hospital, Toon 791-0295, Japan. ${ }^{6}$ Core Research for Evolutional Science and Technology, Japan Science and Technology Agency, Kawaguchi 332-0012, Japan. Correspondence and requests for materials should be addressed to K.K.

(email: kazuto@fmu.ac.jp).
} 
$\mathrm{F}$ lexible switching of behaviours in response to changed surroundings is essential for adaptation to the environment and survival. This behavioural flexibility depends on the integrative function of the prefrontal cortex including the orbitofrontal, prelimbic and infralimbic cortical areas ${ }^{1-4}$. It also requires the dorsomedial striatum $(\mathrm{DMS})^{5-7}$ that receives dense innervations from the prefrontal cortex ${ }^{8,9}$. Multiple neurotransmitter systems that influence the neural circuitry linking the prefrontal cortex and DMS modulate behavioural flexibility ${ }^{10-12}$. In addition, severe deficits in cognitive flexibility are associated with certain neuropsychiatric diseases, such as schizophrenia and attention deficit hyperactivity disorder ${ }^{13-17}$.

Cholinergic interneurons in the striatum, known as tonically active neurons, respond to a variety of stimuli related to reward prediction, attention and context recognition during learning processes $^{18-20}$. Striatal cholinergic interneurons also influence dopamine-dependent motor behaviour and reward-related learning ${ }^{21,22}$. Acetylcholine efflux in the striatum increases in the phase of behavioural switching ${ }^{23,24}$. Previous pharmacologic studies have suggested the role of striatal acetylcholine in the facilitation of behavioural flexibility ${ }^{25-28}$. Infusion of a single dose of the nonspecific muscarinic acetylcholine receptor antagonist scopolamine into the DMS impairs reversal learning based on response discrimination ${ }^{25}$. Infusion of an $\mathrm{M}_{1}$-type muscarinic receptor antagonist, such as pirenzepine or muscarinic toxin 7 , inhibits place reversal learning ${ }^{26,27}$, whereas infusion of an $\mathrm{M}_{4^{-}}$ type muscarinic receptor antagonist, muscarinic toxin 3 , does not affect the same learning paradigm ${ }^{27}$. Administration of oxotremorine sesquifumarate, which is an $\mathrm{M}_{2} / \mathrm{M}_{4}$ muscarinic agonist with a higher affinity to presynaptic $\mathrm{M}_{2}$ muscarinic receptor localized in the striatal cholinergic interneurons, suppresses acetylcholine efflux and disturbs reversal learning ${ }^{28}$, but it also possesses affinity for $\mathrm{M}_{4}$ muscarinic receptor ${ }^{29}$. Because of the complexity in dose responses and the broad spectrum of drug affinity to the receptors, the pharmacologic evidence is limited for determining the exact role of striatal cholinergic interneurons in behavioural flexibility.

Here we address the role of cholinergic interneurons in the striatum in reversal and extinction learning based on place discrimination by inducing selective elimination of this interneuronal type in rats with immunotoxin (IT)-mediated cell targeting ${ }^{30,31}$. IT targeting of cholinergic interneurons from the DMS, but not from the dorsolateral striatum (DLS), results in enhanced reversal and extinction learning, normally showing the acquisition of spatial discrimination. This enhancement is blocked by the infusion of a non-selective muscarinic receptor agonist into the DMS either in the acquisition, reversal or extinction phase. In addition, short hairpin RNA (shRNA)mediated gene silencing of $\mathrm{M}_{4}$ muscarinic receptor mimics the place reversal learning promoted by cholinergic targeting, whereas gene silencing of $M_{1}$ muscarinic receptor does not alter reversal learning performance. Our data, in contrast to the previous pharmacological evidence, demonstrate that the DMS cholinergic interneurons possess an inhibitory role in behavioural flexibility, predominantly through the $\mathrm{M}_{4}$ muscarinic receptor. This cholinergic function appears to stabilize the acquired reward contingency and to suppress the response switch to changed contingency.

\section{Results}

IT targeting of striatal cholinergic interneurons. To investigate the role of striatal cholinergic interneurons in behavioural flexibility, we performed selective elimination of these interneurons in the dorsal striatum by IT targeting technology $y^{30,31}$. In this technology, transgenic ( $\mathrm{Tg}$ ) animals are generated that express human interleukin-2 receptor $\alpha$-subunit (IL-2R $\alpha$ ) under the control of a cell type-specific promoter. These animals are then injected with a recombinant IT containing variable regions of anti-IL-2R $\alpha$ monoclonal antibody fused to a bacterial exotoxin fragment, which results in the removal of target cell types bearing human IL-2R $\alpha$. To induce IL-2R $\alpha$ gene expression in cholinergic neurons, we produced a transgene construct, in which the gene cassette containing IL- $2 \mathrm{R} \alpha$ fused to a variant of enhanced yellow fluorescent protein (mVenus) ${ }^{32}$ and followed by the polyadenylation signal was introduced into the initiation codon site of the gene encoding choline acetyltransferase (ChAT) in a bacterial artificial chromosome clone (Fig. 1a). Using the microinjection technique, we generated multiple independent $\mathrm{Tg}$ rat founders. One strain, termed ChAT-IL-2R $\alpha / m$ Venus-4-53, expressed the IL-2R $\alpha / \mathrm{mVenus}$ gene in the striatum (Fig. 1b). Double immunofluorescence histochemistry detected the majority of mVenus-positive signals localized in ChAT-positive neurons (Fig. 1c), suggesting cholinergic neuron-specific expression of the transgene driven by the ChAT gene promoter.

Rats were injected unilaterally with IT solution ( $5 \mathrm{ng}$ per site $\times 6$ sites) or phosphate-buffered saline (PBS) into the dorsal striatum via stereotaxic surgery (Fig. 1d). Seven days after IT injection, brains were processed into sections through the striatum for histologic analysis. Immunohistochemical staining of the sections showed a loss of ChAT-positive neurons only in the IT-injected Tg rats (Fig. 1e) and the number of ChAT-positive neurons in the dorsal striatum was counted (Fig. If and Supplementary Fig. 1 for region of interest used for cell counts). One-way analysis of variance (ANOVA) indicated a significant difference among the rat groups $\left(F_{(3,12)}=26.562, P=0.000\right)$, showing a marked reduction in the IT-injected Tg rats compared with the PBS- or IT-injected non-Tg (non-Tg) rats or the PBSinjected Tg rats $(P<0.05$, Bonferroni method). The cell loss was restricted in the dorsal striatum and did not extend to other brain regions including cholinergic neurons, such as the prefrontal cortex, nucleus accumbens and nucleus basalis magnocellularis (Supplementary Fig. 2). Cresyl violet staining of the sections showed no grossly visible damage of brain tissue, and stainings with other specific striatal cell markers displayed intact medium spiny neurons and GABAergic interneurons (Supplementary Fig. 3). In addition, immunohistochemical analysis for axon terminals confirmed the normal distribution of glutamatergic and dopaminergic terminals innervating the dorsal striatum (Supplementary Fig. 4). These data supported the selective, efficient elimination of cholinergic interneurons in the dorsal striatum of the IT-injected Tg animals.

Striatal cholinergic targeting enhances reversal learning. To evaluate behavioural switching in rats lacking striatal cholinergic interneurons, we conducted reversal learning by using a place discrimination task with a modified cross maze ${ }^{27}$ (see Fig. 1g). In the acquisition phase, one choice arm (correct arm) of the maze was reinforced by $30 \%$ sucrose solution, and another choice arm (incorrect arm) was not. Rats entered the maze from the start arm and were required to select the correct arm. In the reversal learning phase, the positions of the correct and incorrect arms were inverted to test the rat's ability to select the correct arm in response to the changed situation. When the $\mathrm{Tg}$ and non-Tg rats were given a bilateral intrastriatal injection of IT solution, the correct choice ratio in the acquisition phase showed a similar gradual increase between groups (Fig. 1h; group effect, $F_{(1,14)}=1.152, P=0.301$; block effect, $F_{(7,98)}=6.289, P=0.000$, interaction, $F_{(7,98)}=1.099, P=0.370$, two-way ANOVA), whereas in the reversal learning phase the increment in the correct choice ratio in the $\mathrm{Tg}$ rats was accelerated compared with that in the 
a

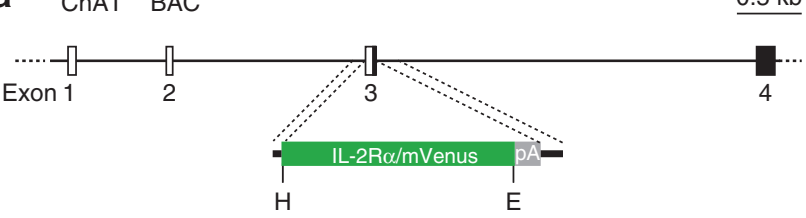

C
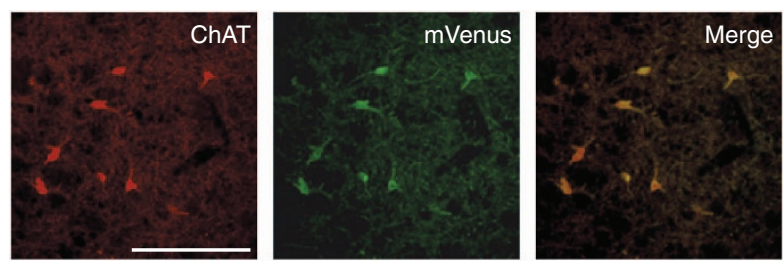

b
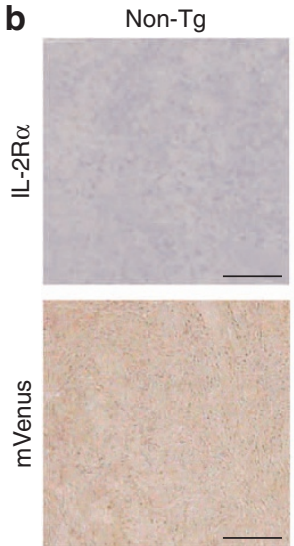
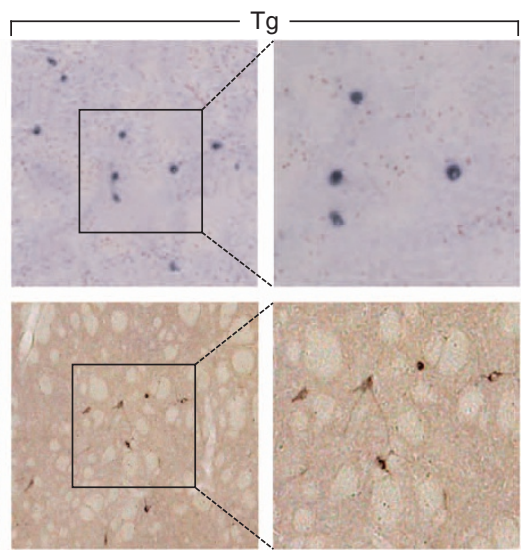

d

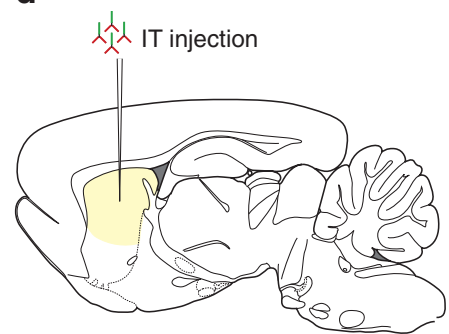

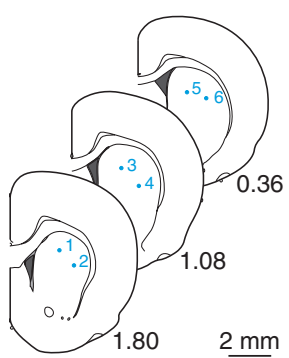

e

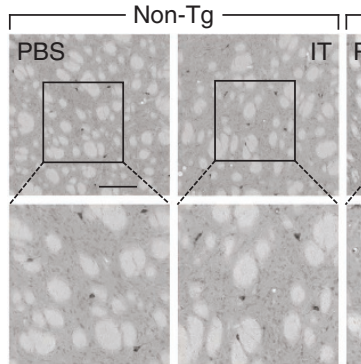

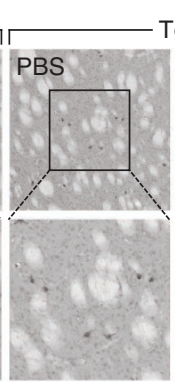

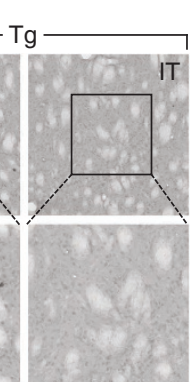

f

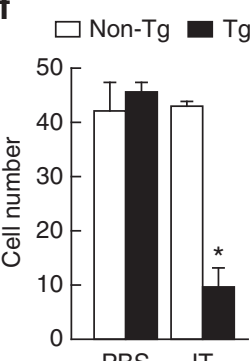

g

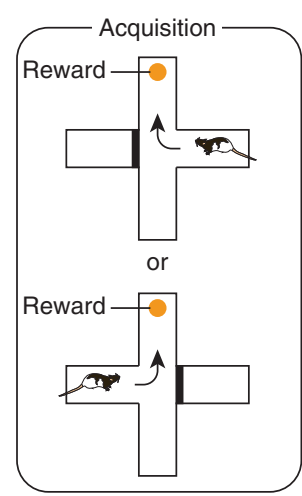

h

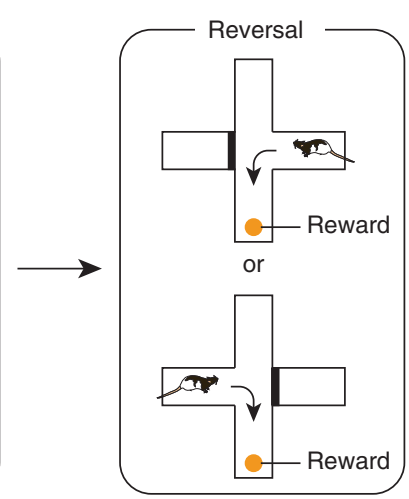

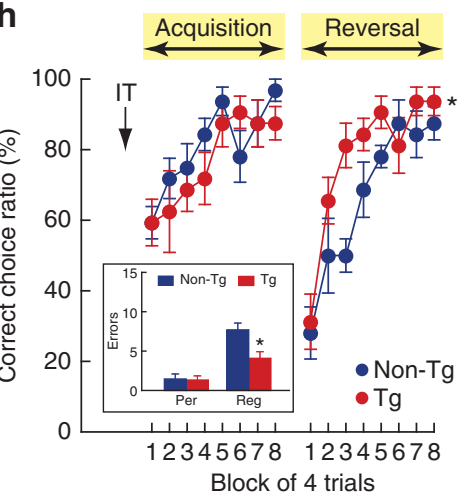

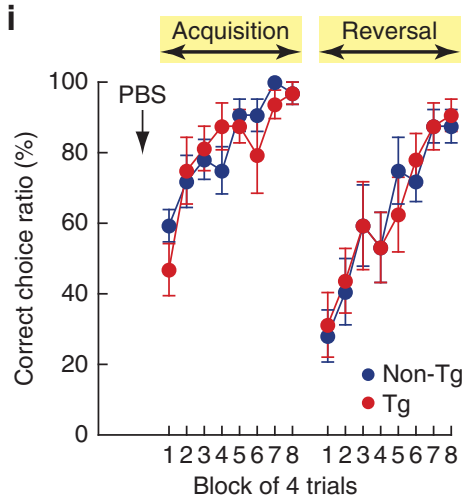

Figure 1 | Selective targeting of striatal cholinergic interneurons results in enhancement of reversal learning. (a) Transgene construct for generating rats expressing the human IL-2R $\alpha / m$ Venus fusion protein under the control of the ChAT gene promoter. Exons 1-4 of the rat ChAT gene are shown. Open and closed boxes indicate the $5^{\prime}$-noncoding region and coding regions, respectively. Restriction enzyme abbreviations: $\mathrm{E}, \mathrm{EcoRI} ; \mathrm{H}, \mathrm{HindlII}$. pA; polyadenylation signal from the SV40 early gene. (b) Expression of IL-2R $\alpha / m V e n u s$ products in the dorsal striatum of Tg rats revealed by in situ hybridization analysis for IL-2R $\alpha$ sequence (upper panel) and immunohistochemical staining for mVenus (lower panel). Scale bars, $200 \mu \mathrm{m}$.

(c) Double immunofluorescence histochemistry for mVenus and ChAT. mVenus- and ChAT-positive signals are shown in green and red, respectively. In a merged image, green signals overlap with red signals, and thus emit yellow signals. Scale bar, $200 \mu \mathrm{m}$. (d) Intrastriatal injection by stereotaxic surgery. Right panel indicates coordinates for the injection (sites 1-6). The anteroposterior coordinates ( $\mathrm{mm}$ ) from bregma are shown. Scale bar, $2 \mathrm{~mm}$.

(e) Immunohistochemical staining of the striatal section for ChAT, showing selective loss of ChAT-positive neurons in the IT-injected Tg rats. Lower panels are fourfold magnified view of the squares in the upper panels. Scale bar, $200 \mu \mathrm{m}$. (f) Cell counts of ChAT-positive cells. $n=4$ per group. ${ }^{\star} P<0.05$, relative to the PBS- and IT-injected non-Tg and the PBS-injected Tg rats (Bonferroni method). (g) Strategy of a place discrimination task for evaluating the performance of reversal learning. The entrance to one of the arms in the cross maze was prevented. The positions of the start arms were changed in a pseudorandom order. (h,i) Place discrimination task of the rats bilaterally injected with IT solution (h) or PBS (i) into the dorsal striatum. The performance in the acquisition and reversal learning phases were tested. $n=8$ per group. ${ }^{*} P<0.05$, relative to the IT-injected non-Tg rats (Bonferroni method). An inset shows the number of perseverative (Per) and regressive (Reg) errors in the reversal learning phase in the IT-injected rats. ${ }^{\star} P<0.01$, relative to the non-Tg rats (Student's $t$-test). Data are presented as mean \pm s.e.m.

non-Tg rats (Fig. 1h; group effect, $F_{(1,14)}=6.194, P=0.026$; block effect, $F_{(7,98)}=24.187, P=0.000$, interaction, $F_{(7,98)}=0.778$, $P=0.393$, two-way ANOVA). In contrast, when rats received the bilateral PBS injection, the correct choice ratio was increased similarly between the $\mathrm{Tg}$ and non-Tg rats in both the acquisition phase (Fig. 1i; group effect, $F_{(1,14)}=0.276, P=0.608$; block effect, $F_{(7,98)}=11.233, P=0.000$, interaction, $F_{(7,98)}=0.956, P=0.468$, two-way ANOVA) and reversal phase (Fig. 1i; group effect, $F_{(1,14)}=0.002, P=0.963$; block effect, $F_{(7,98)}=18.867, P=0.000$, interaction, $F_{(7,98)}=0.339, P=0.934$, two-way ANOVA). These 
data indicate that the elimination of striatal cholinergic interneurons in the IT-injected $\mathrm{Tg}$ rats resulted in enhanced spatial, reversal learning, normally sparing the acquisition of learning.

A previous study categorized errors in the phase of reversal learning into 'perseverative errors', defined as incorrect choices that occur until an animal first selects the new correct arm, and 'regressive errors', defined as incorrect choices that occur afterwards ${ }^{27}$. These two categories of errors were used to classify the errors that occurred in the reversal learning phase in the IT-injected rats (Fig. 1h). The number of perseverative errors was not different between the non- $\mathrm{Tg}$ and $\mathrm{Tg}$ rats that received the IT injection, whereas the number of regressive errors was significantly lower in the $\mathrm{Tg}$ rats compared with the non- $\mathrm{Tg}$ rats $\left(t_{(14)}=3.145, P=0.007\right)$. The data suggest that the enhanced reversal learning in the injected $\mathrm{Tg}$ rats was not attributable to the change in perseveration of the previously learned strategy but to an increased ability to learn the new strategy.

To explore whether enhanced reversal learning in the IT-injected $\mathrm{Tg}$ rats was caused by targeting of the cholinergic interneurons in the DMS or DLS, rats were given a local injection of IT solution into each subregion via stereotaxic surgery (Fig. 2a). The local IT injection achieved selective targeting of cholinergic interneurons in the DMS or DLS in the Tg rats (Fig. 2b), and the number of ChAT-positive neurons in region of interest in each subregion was counted (Fig. 2c and Supplementary Fig. 5 for cell counts in the subregions). Oneway ANOVA indicated a significant difference among the groups $\left(F_{(3,12)}=16.821, P=0.000\right.$ for DMS injection; $F_{(3,12)}=31.409$, $P=0.000$ for DLS injection), showing a significant reduction in the corresponding subregion in $\mathrm{Tg}$ rats compared with each of the other three groups $(P<0.05$, Bonferroni method).

The rats were given bilateral, local IT injections into the DMS or DLS and then tested for reversal learning by using place discrimination (Supplementary Fig. 6. for cell ablation range). The ablation of the DMS cholinergic interneurons in $\mathrm{Tg}$ rats showed a gradual increase in the correct choice ratio in the acquisition phase in a similar fashion to the non-Tg controls (Fig. 2d; group effect, $F_{(1,13)}=1.163, P=0.300$; block effect, $F_{(7,91)}=18.269, P=0.000$, interaction, $F_{(7,91)}=1.152, P=0.338$, two-way ANOVA) and promoted an increase in the ratio in the reversal learning phase as compared with the controls (Fig. 2d; group effect, $F_{(1,13)}=5.044, P=0.043$; block effect, $F_{(7,91)}=33.287, P=0.000$, interaction, $F_{(7,91)}=1.163, P=0.332$, two-way ANOVA). Although the number of perseverative errors did not vary between the two rat groups, the number of regressive errors was significantly decreased in the Tg rats compared with the non-Tg rats (Fig. $2 \mathrm{~d} ; t_{(13)}=2.170, P=0.049$ ). In contrast, the Tg rats lacking the DLS cholinergic interneurons exhibited a normal performance in the acquisition and reversal phases (Fig. 2e; group effect, $F_{(1,14)}=1.776, P=0.204$; block effect, $F_{(7,98)}=21.829, P=0.000$, interaction, $F_{(7,98)}=1.777, P=0.100$, for acquisition; group effect, $F_{(1,14)}=0.294, P=0.596$; block effect, $F_{(7,98)}=26.369, \quad P=0.000$, interaction, $F_{(7,98)}=1.421$, $P=0.205$, for reversal learning, two-way ANOVA). Therefore, enhanced reversal learning was induced by elimination of cholinergic interneurons in the DMS but not in the DLS, supporting the importance of the DMS in behavioural flexibility $^{5-7}$.

Cholinergic targeting accelerates extinction learning. Reversal learning is generally considered to involve both extinction of a previously learned response and acquisition of a newly reinforced response. The enhanced reversal learning in the rats lacking striatal cholinergic interneurons may be associated with the alteration in extinction learning. To test this possibility, we conducted extinction learning in the place discrimination task with the modified cross maze. In the acquisition phase, rats were required to choose the correct arm in the maze. In the extinction learning phase, the rewards were removed from the arm and the animals were tested for approach into the arms that had held a reward in the previous phase. We first explored the performance of extinction learning with rats in which striatal cholinergic interneurons were deleted in the whole region of the dorsal striatum. When the rats were given the intrastriatal injection of IT solution bilaterally, the previously rewarded arm choice ratio in the extinction phase decreased faster in the $\mathrm{Tg}$ rats than in the non-Tg controls (Fig. 3a; group effect, $F_{(1,11)}=18.664, P=0.001$; block effect, $F_{(7,77)}=18.425, P=0.000$, interaction, $F_{(7,77)}=1.484$, $P=0.186$, two-way ANOVA). In contrast, when the rats received PBS injection, the choice ratio declined similarly between the two rat groups in the same phase (Fig. $3 b$; group effect, $F_{(1,14)}=2.301$, $P=0.152$; block effect, $F_{(7,98)}=25.914, P=0.000$, interaction, $F_{(7,77)}=0.985, P=0.447$, two-way ANOVA).

In the extinction learning phase, the rats initially selected the previously rewarded arm, but then they gradually switched to selecting the previously non-rewarded arm or neither arm. The ratio of selecting the previously non-rewarded arm gradually increased in the early stage of trials in both $\mathrm{Tg}$ and non-Tg rats treated with IT, but it decreased faster in the Tg rats than in the non-Tg rats in the middle stage of trials. Both rat groups had an increased tendency to select neither arm, and the increase in the $\mathrm{Tg}$ rats was accelerated compared with the non-Tg group (see Supplementary Fig. 7). These observations support enhanced extinction learning in rats lacking striatal cholinergic interneurons.

Next, we tested the efficacy of targeting of cholinergic interneurons in the DMS or DLS on extinction learning. Rats were given a local, bilateral injection of IT solution into the two subregions and subjected to the place discrimination task. The ablation of the DMS cholinergic interneurons in $\mathrm{Tg}$ rats accelerated the reduction in the previously rewarded arm choice ratio in the extinction phase as compared with the non-Tg controls (Fig. 3c; group effect, $F_{(1,12)}=2.475, P=0.142$; block effect, $F_{(7,84)}=16.606, P=0.000$; interaction, $F_{(7,84)}=2.754$, $P=0.013$, two-way ANOVA), whereas the Tg rats lacking the DLS cholinergic interneurons displayed the normal extinction learning (Fig. 3d; group effect, $F_{(1,9)}=0.034, P=0.859$; block effect, $F_{(7,63)}=15.769, P=0.000$, interaction, $F_{(7,63)}=0.321$, $P=0.942$, two-way ANOVA). Thus, the elimination of DMS cholinergic interneurons improved the performance of extinction learning, suggesting that enhanced reversal learning caused by cholinergic elimination is associated, at least in part, with the improvement of extinction learning.

Muscarinic restoration of altered behavioural flexibility. To confirm enhanced behavioural flexibility by striatal cholinergic targeting, we examined whether the altered learning performance in the IT-injected $\mathrm{Tg}$ rats could be restored by infusion of a nonspecific muscarinic receptor agonist oxotremorine methiodide (OxoM). Initially, the altered reversal learning by cholinergic ablation was tested for the restoration by the muscarinic activation. Rats were given a bilateral injection of the IT solution into the DMS and received a bilateral infusion of OxoM solution (4.0 ng per site) or PBS into the same subregion prior to each daily trial either in the acquisition or reversal phase. When the drug infusion was carried out in the acquisition phase, the PBSinfused $\mathrm{Tg}$ rats exhibited enhanced performance of reversal learning but the OxoM-infused $\mathrm{Tg}$ rats showed performance comparable with the non-Tg control levels (Fig. 4a; group effect, 
a
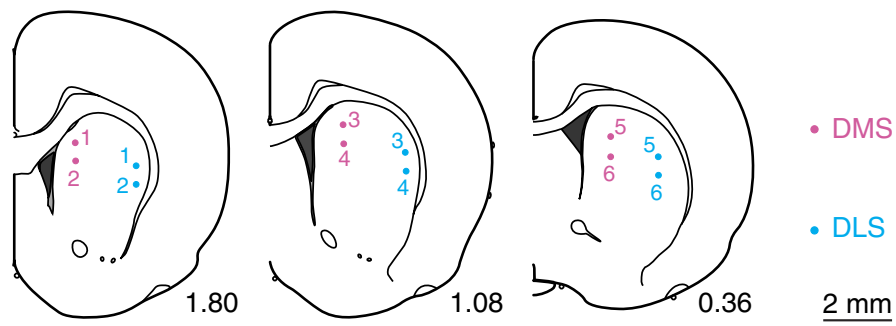

b

DMS injection

DLS injection
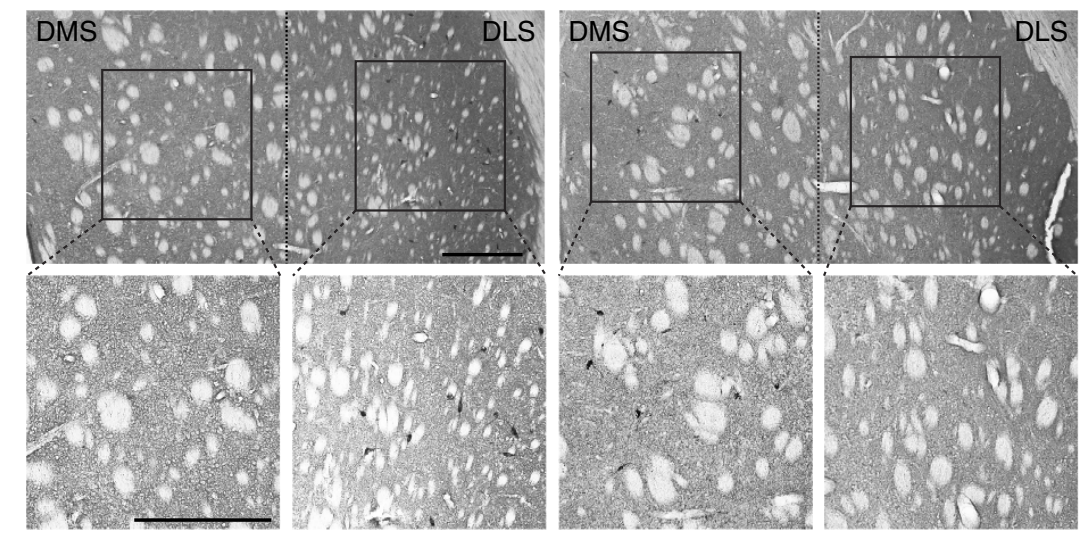

C

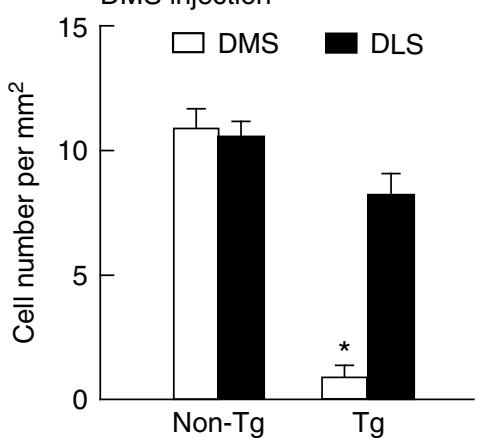

DLS injection

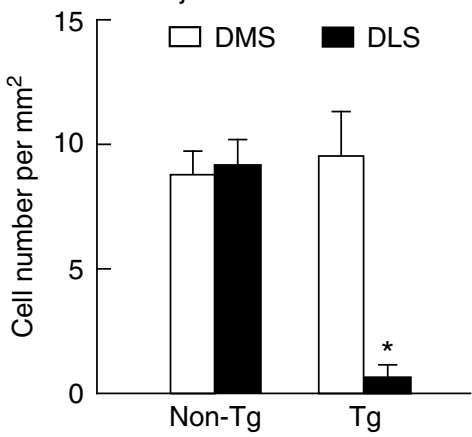

d

DMS injection

e

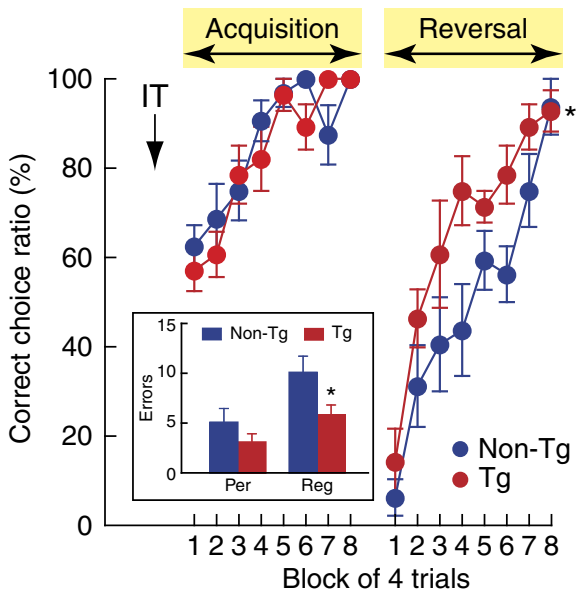

DLS injection

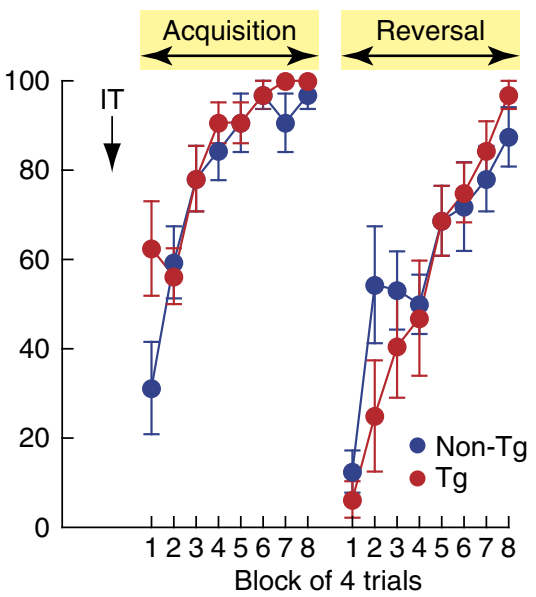

Figure 2 | Targeting of the DMS cholinergic interneurons enhances reversal learning performance. (a) Coordinates for injection of IT solution into the DMS or DLS by stereotaxic surgery. The anteroposterior coordinates ( $\mathrm{mm}$ ) from bregma are shown. Scale bar, $2 \mathrm{~mm}$. (b) Representative images of ChAT immunohistochemical staining of striatal sections prepared from the IT-injected Tg rats, indicating selective loss of ChAT-positive neurons in the DMS or DLS. Lower panels are fourfold magnified view of the squares in the upper panels. Scale bar, $400 \mu \mathrm{m}$. (c) Cell counts of ChAT-positive cells in the region of interest in subregions. $n=4$ per group. ${ }^{\star} P<0.05$, relative to other three groups (Bonferroni method). (d,e) Place discrimination task of the rats injected bilaterally with IT solution into the DMS (d) or DLS (e). The performance in the acquisition and reversal learning phases were tested. DMS injection: $n=8$ for non-Tg, $n=7$ for Tg; DLS injection: $n=8$ for non-Tg/Tg. ${ }^{*} P<0.05$, relative to the IT-injected non- $\mathrm{Tg}$ rats (Bonferroni method). An inset shows the number of perseverative (Per) and regressive (Reg) errors in the reversal learning phase in the IT-injected rats. ${ }^{\star} P<0.05$, relative to the non-Tg rats (Student's $t$-test). Data are presented as mean \pm s.e.m. 

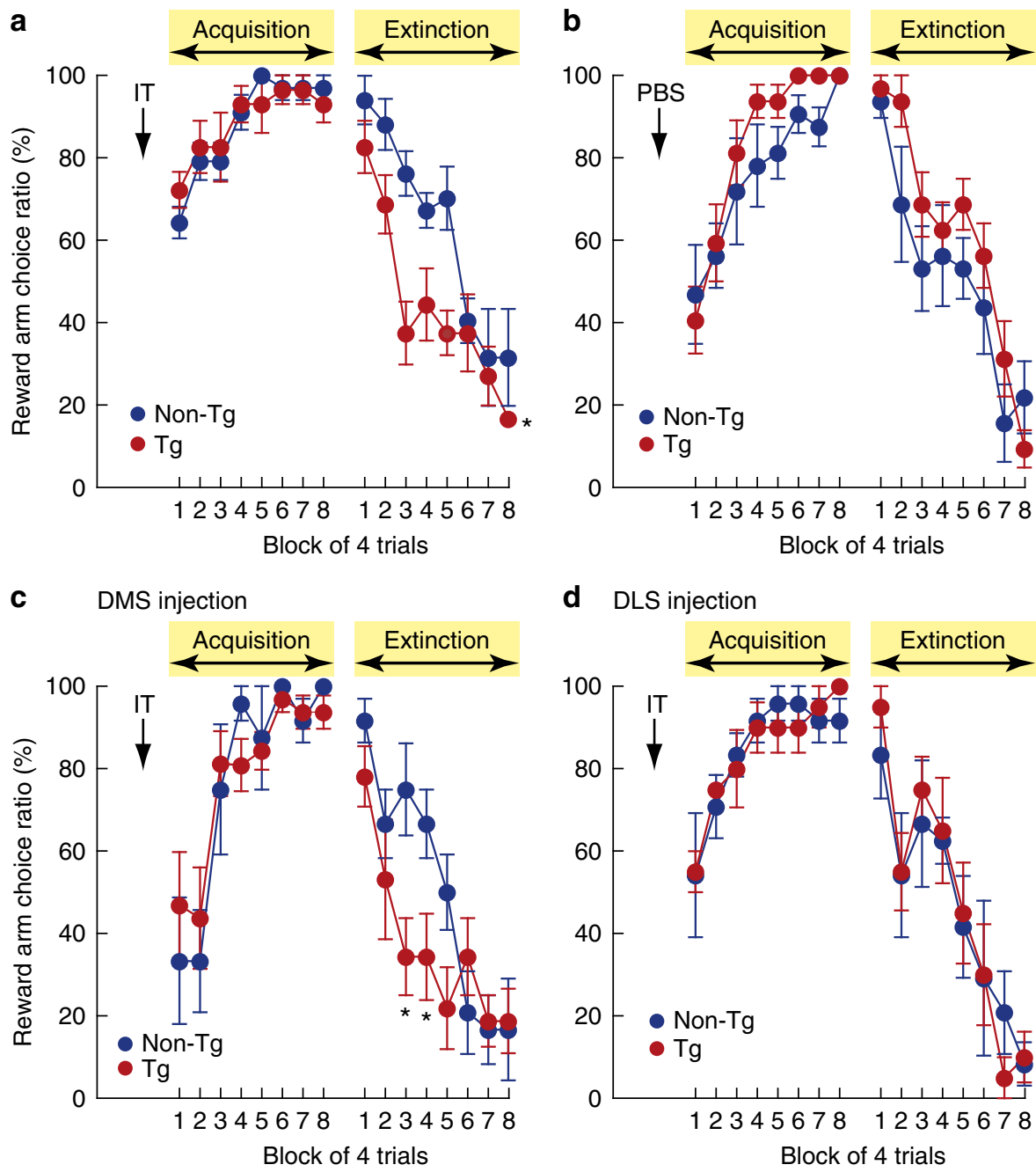

d DLS injection

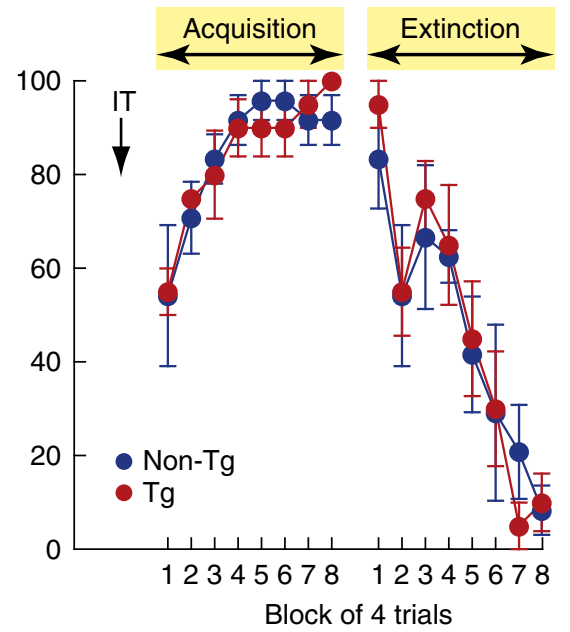

Figure 3 | Elimination of the DMS cholinergic interneurons accelerates extinction learning performance. (a,b) Place discrimination performance of the rats injected bilaterally with IT solution (a) or PBS (b) into the dorsal striatum. The performance in the acquisition and extinction learning phases were tested. IT injection: $n=7$ for non- $\mathrm{Tg}, n=6$ for Tg; PBS injection: $n=8$ for non- $\mathrm{Tg} / \mathrm{Tg}$. ${ }^{\star} P<0.05$, relative to the non- $\mathrm{Tg}$ rats (Bonferroni method). (c,d) Learning performance of the rats that received bilateral IT injection into the DMS (c) or DLS (d). DMS injection: $n=6$ for non-Tg, $n=8$ for Tg; DLS injection: $n=6$ for non-Tg, $n=5$ for $\mathrm{Tg}$. ${ }^{\star} P<0.05$, relative to the non-Tg rats (Bonferroni method). Data are presented as mean \pm s.e.m.

$F_{(3,28)}=5.636, P=0.004$; block effect, $F_{(7,196)}=64.896, P=0.000$; interaction, $F_{(21,196)}=1.992, P=0.008$, two-way ANOVA). When the drug was infused in the reversal phase, the OxoM-infused $\mathrm{Tg}$ rats displayed reversal learning performance similar to the nonTg controls (Fig. 4b; group effect, $F_{(3,28)}=3.008, P=0.047$; block effect, $F_{(7,196)}=65.971, P=0.000$; interaction, $F_{(21,196)}=2.152$, $P=0.004$, two-way ANOVA).

Subsequently, the influence of muscarinic activation on the changed extinction learning was tested. When the drug infusion was performed in the acquisition phase, the PBS-infused Tg rats exhibited accelerated performance of extinction learning but the extinction performance of the OxoM-infused $\mathrm{Tg}$ was normalized (Fig. 4c; group effect, $F_{(3,23)}=5.446, P=0.006$; block effect, $F_{(7,161)}=58.190, P=0.000$; interaction, $F_{(21,161)}=1.690$, $P=0.037$, two-way ANOVA). When the drug was infused in the extinction phase, the OxoM-infused Tg rats exhibited extinction performance resembling the non-Tg controls (Fig. 4d; group effect, $F_{(3,23)}=2.834, P=0.061$; block effect, $F_{(7,161)}=68.867$, $P=0.000 ;$ interaction, $F_{(21,161)}=1.854, \quad P=0.017$, two-way ANOVA). These results demonstrate that the altered performance of reversal and extinction learning in rats lacking the DMS cholinergic interneurons can be reversed to normal levels by muscarinic activation of the striatum either in the acquisition or reversal/extinction phase.

Locomotor activity in rats lacking cholinergic interneurons. To check the impact of striatal cholinergic elimination on spontaneous locomotion, rats were injected bilaterally with IT solution or PBS into the entire dorsal striatum and analysed for locomotion in the open field test. The IT-injected Tg rats did not show any change in locomotor activity, displaying normal habituation to the environment (Fig. 5a; group effect, $F_{(3,26)}=1.211, P=0.326$; block effect, $F_{(5,130)}=40.513, P=0.000$; interaction, $F_{(15,130)}=0.551, \quad P=0.906$, two-way ANOVA). Average velocity of ambulation in the IT-injected $\mathrm{Tg}$ rats was indistinguishable from that in other rat groups (Fig. 5b; $F_{(3,26)}=0.227, P=0.877$, one-way ANOVA). The results indicate that spontaneous locomotion was apparently normal in the $\mathrm{Tg}$ rats, suggesting that altered performance in reversal/extinction learning by cholinergic elimination was not relevant to changes in general motor behaviour in these animals. 
a

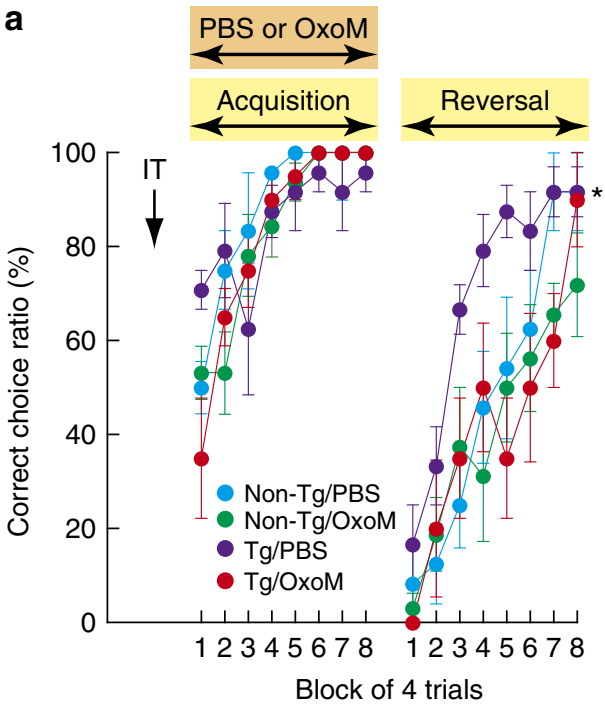

C

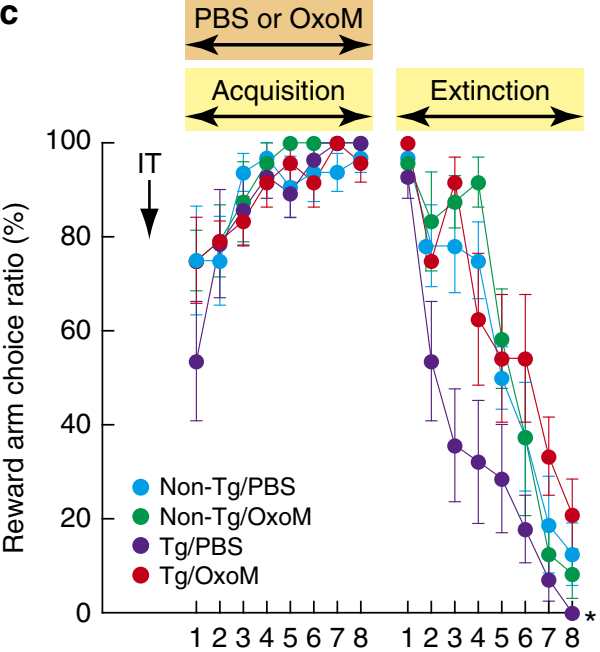

Block of 4 trials b

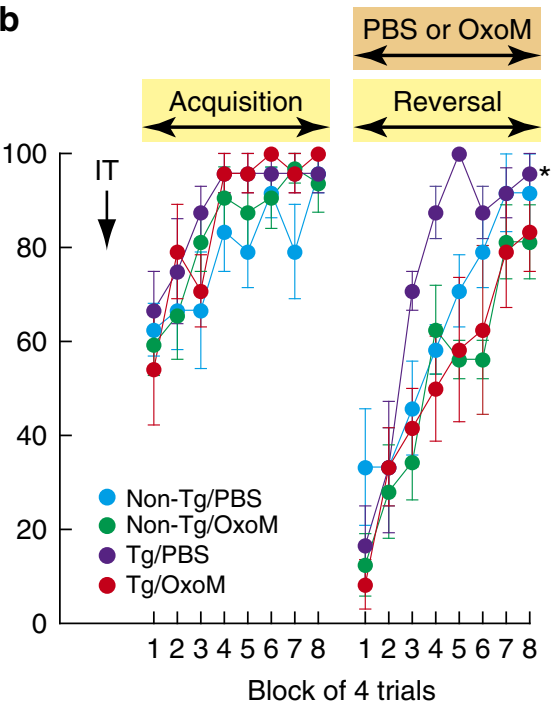

d

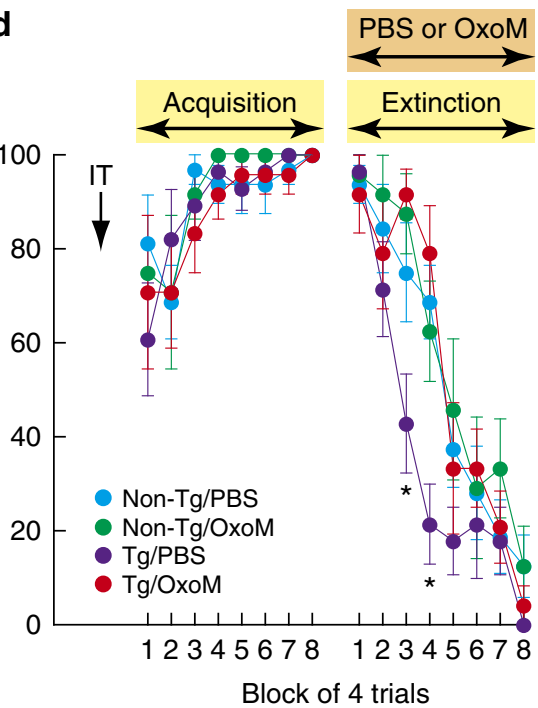

Figure 4 | Rescue of the altered reversal and extinction learning by muscarinic activation in the striatum. (a,b) Performance of reversal learning of the IT-injected rats that were given bilaterally infusion of OxoM or PBS into the DMS in either the acquisition (a) or reversal learning (b) phase. $n=10$ for non-Tg/PBS, $n=8$ for non-Tg/OxoM, $n=8$ for Tg/PBS, $n=6$ for Tg/OxoM. ${ }^{\star} P<0.05$, relative to any of the other three groups (Bonferroni method). (c,d) Performance of extinction learning of the IT-injected rats that received bilateral infusion of OxoM or PBS into the DMS in either the acquisition (c) or extinction learning (d) phase. $n=10$ for non-Tg/PBS, $n=6$ for non- Tg/OxoM, $n=8$ for Tg/PBS, $n=6$ for Tg/OxoM. ${ }^{\star} P<0.05$, relative to any of the other three groups (Bonferroni method). Data are presented as mean \pm s.e.m.
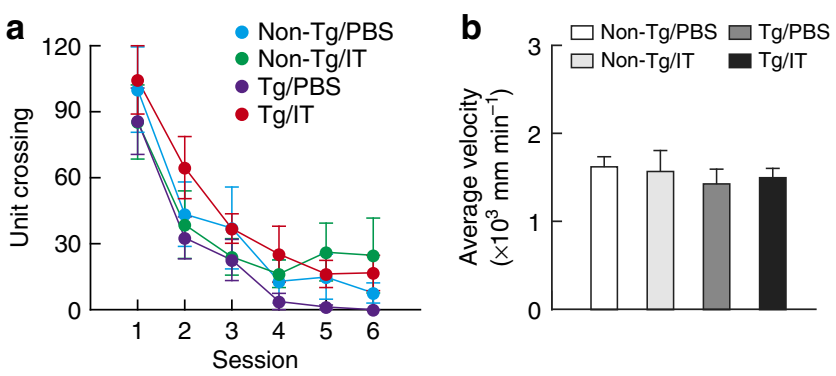

Figure 5 | Spontaneous locomotion in rats lacking striatal cholinergic interneurons. Non- $\mathrm{Tg}$ and $\mathrm{Tg}$ rats received a bilateral injection of IT solution or PBS into the dorsal striatum and were placed in the open field. (a) Time course of unit crossings during a 10-min session. (b) Average velocity of ambulation. $n=6$ for non-Tg/PBS, $n=8$ for other three groups. Data are presented as mean \pm s.e.m.
$\mathrm{M}_{4}$ receptor knockdown increases reversal learning. Previous pharmacologic studies have suggested that striatal acetylcholine facilitates behavioural flexibility ${ }^{25-28}$. This cholinergic function is reported to be transmitted through $\mathrm{M}_{1}$ muscarinic receptor ${ }^{26,27}$. In contrast to this previous pharmacologic view, the selective elimination of striatal cholinergic interneurons resulted in enhanced reversal and extinction learning, suggesting the role of these interneurons in the suppression of behavioural flexibility. $\mathrm{M}_{4}$ muscarinic receptor is localized in the majority of striatal projection neurons ${ }^{33,34}$ and its allosteric modulation antagonizes hyperactivity in response to psychostimulant ${ }^{35,36}$. Although an $\mathrm{M}_{4}$-type muscarinic receptor antagonist is reported to have no effect on reversal learning ${ }^{27}$, the function of this receptor subtype has not been fully characterized. There is the possibility that the inhibitory role of striatal cholinergic interneurons may be predominantly mediated via $\mathrm{M}_{4}$ muscarinic receptor.

To test this possibility, we investigated the efficacy of knockdown of $\mathrm{M}_{4}$ muscarinic receptor gene for reversal learning 
of place discrimination. We designed six different shRNA sequences against rat $M_{4}$ muscarinic receptor gene $\left(M_{4}\right.$ shRNA-1 to -6) for lentiviral vector-mediated expression (see Supplementary Fig. 8 for shRNA expression vector and its sequences) and preliminarily checked their knockdown efficacy in cultured cells (Supplementary Fig. 9). Because $\mathrm{M}_{4}$ shRNA-3 produced the greatest knockdown effect, we used it for the in vivo experiments. Long Evans rats were given a unilateral injection of lentiviral vector encoding $\mathrm{M}_{4}$ shRNA-3 or non-target control sequence, together with green fluorescent protein (GFP) gene cassette $\left(3 \times 10^{8}\right.$ transduction units $\mathrm{ml}^{-1}$, three sites). Three weeks later, brain sections through the striatum were processed and analysed by histologic examinations. GFP immunohistochemistry showed gene transduction into the DMS subregion by lentiviral vectors encoding $\mathrm{M}_{4}$ or control shRNA (Fig. 6a), and double immunostaining for GFP and NeuN indicated the transduction of a large number of neurons in both vectorinjected sides (Fig. 6b; 65.1\% in $\mathrm{M}_{4}$ shRNA and $66.3 \%$ in the control). Cresyl violet staining of striatal sections confirmed no visible tissue damage in these injection areas (Fig. 6c). On the same day, the DMS region was dissected out, and messenger RNA (mRNA) level in the tissue was measured by quantitative reverse transcription PCR analysis (Fig. 6d). The mRNA level for $\mathrm{M}_{4}$ muscarinic receptor was significantly reduced in the side injected with the $\mathrm{M}_{4}$ shRNA vector relative to the side injected with the control vector $\left(70.2 \%\right.$ of the control value, $t_{(10)}=7.933$, $P=0.000)$. In contrast, the mRNA levels for $M_{1}$ muscarinic receptor were not altered between the two sides. The data indicate the specific knockdown of $\mathrm{M}_{4}$ receptor in the DMS by lentiviral vector-mediated shRNA expression.

To evaluate the influence of $\mathrm{M}_{4}$ muscarinic receptor knockdown on reversal learning, rats were injected bilaterally in the DMS with the lentiviral vector and tested 3 weeks later for the place discrimination task. The rats injected with the $\mathrm{M}_{4}$ shRNA vector gradually acquired the place discrimination in a similar manner to the animals injected with the control shRNA vector (Fig. 6e; group effect, $F_{(1,34)}=0.169, P=0.684$; block effect, $F_{(7,238)}=31.880, P=0.000$; interaction, $F_{(7,238)}=1.650, P=0.122$, two-way ANOVA). The performance of reversal learning was significantly increased in the $\mathrm{M}_{4}$ shRNA vector-injected rat group compared with the control group (Fig. 6e; group effect, $F_{(1,34)}=2.983, P=0.093$; block effect, $F_{(7,238)}=60.516, P=0.000$; interaction, $F_{(7,238)}=2.222, P=0.044$, two-way ANOVA). The number of regressive errors was significantly lower in the $M_{4}$ shRNA vector-injected group compared with the control group (Fig. 6e; $t_{(34)}=2.651, P=0.012$ ), but there was no difference in the number of perseverative errors. Therefore, selective knockdown of $\mathrm{M}_{4}$ muscarinic receptor in the DMS resulted in improved reversal learning of place discrimination, supporting that the striatal cholinergic interneurons act to suppress behavioural flexibility, at least partly, through $\mathrm{M}_{4}$ receptor in the DMS.

$M_{1}$ receptor knockdown does not alter reversal learning. To examine the role of $\mathrm{M}_{1}$ muscarinic receptor in reversal learning,
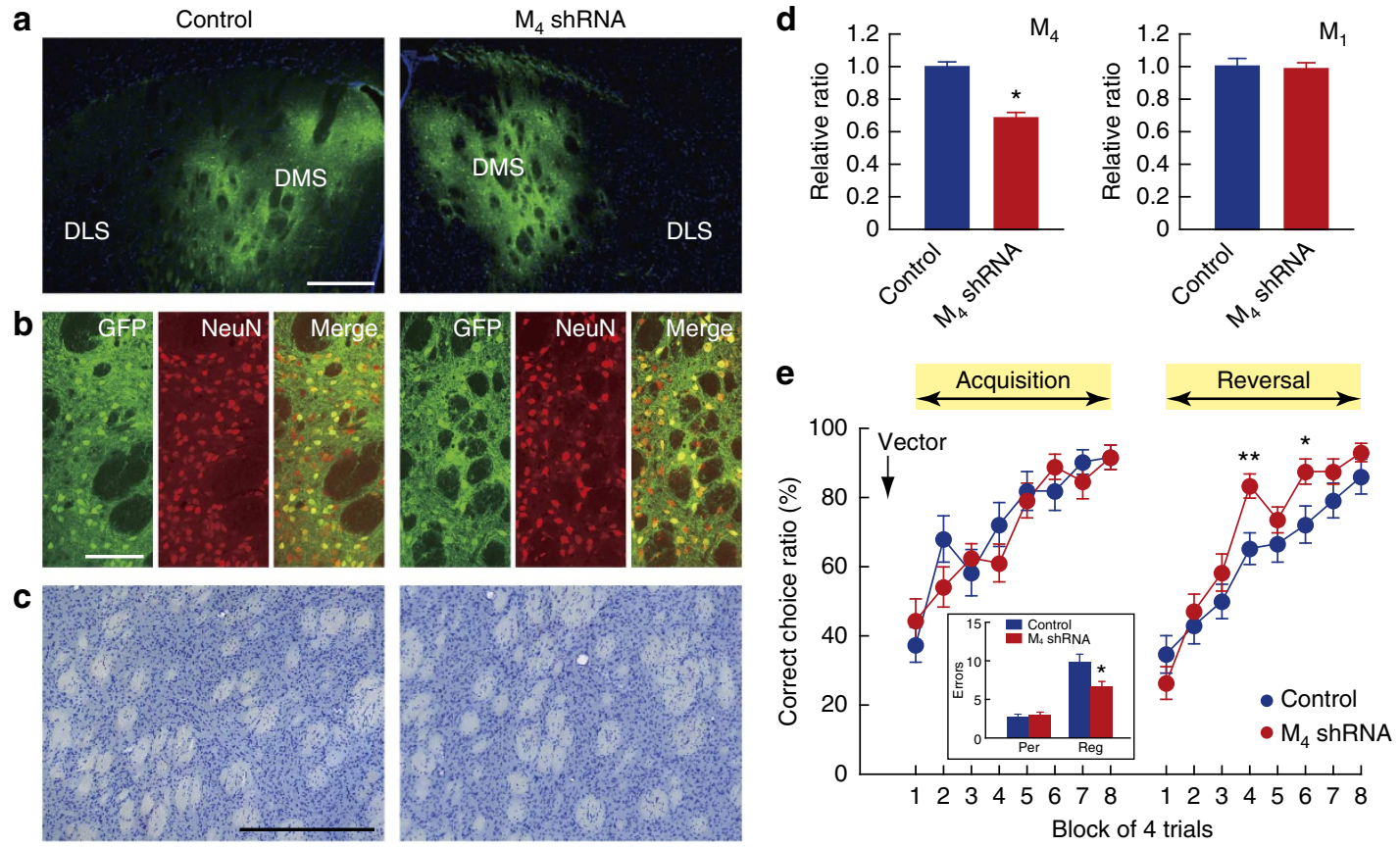

Figure 6 | Gene silencing of $\mathbf{M}_{\mathbf{4}}$ muscarinic receptor in the striatum increases behavioural switching. Long Evans rats were given a unilateral or bilateral injection of lentiviral vectors encoding $\mathrm{M}_{4}$ shRNA and non-target control shRNA in the DMS and 3 weeks later tested for histologic, biochemical and behavioural analyses. (a) Gene transduction areas marked by GFP expression in the rats received unilateral injection of lentiviral vectors. Scale bar, $500 \mu \mathrm{m}$. (b) Efficient transduction of DMS neurons indicated by double immunostaining for GFP and NeuN. Scale bar, $100 \mu \mathrm{m}$. (c), Cresyl violet staining of striatal sections. Scale bar, $500 \mu \mathrm{m}$. (d) $M_{4}$ and $M_{1}$ muscarinic receptor mRNA levels measured by quantitative reverse transcription PCR analysis. Total RNA was extracted from the DMS of the rats that received unilateral injection of vectors and used for quantitative reverse transcription PCR analysis with the primer sets to detect $M_{4}$ and $M_{1}$ muscarinic receptor and $\beta$-actin as an internal control. The relative mRNA levels for the two receptors to $\beta$-actin level were normalized by the average of the values obtained from non-target control shRNA vector-injected side. $n=6$ for each group. ${ }^{\star} P<0.01$, relative to the control group (Student's $t$-test). (e) Performance of reversal learning of rats that received bilateral injection of lentiviral vectors. $n=18$ for each group. ${ }^{\star} P<0.05,{ }^{\star \star} P<0.01$, relative to the control group (Bonferroni method). An inset shows the number of perseverative (Per) and regressive (Reg) errors in the reversal learning phase in the rats injected with $M_{4}$ shRNA vector. ${ }^{\star} P<0.05$, relative to the control group (Student's $t$-test). Data are presented as mean \pm s.e.m. 
a
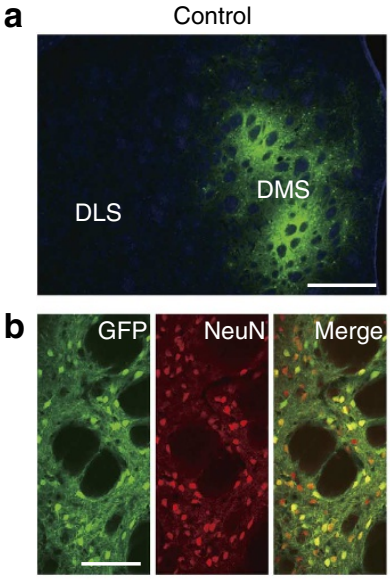

c

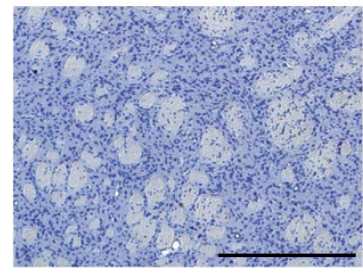

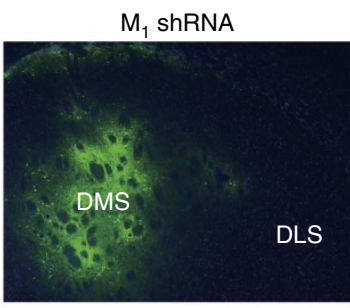
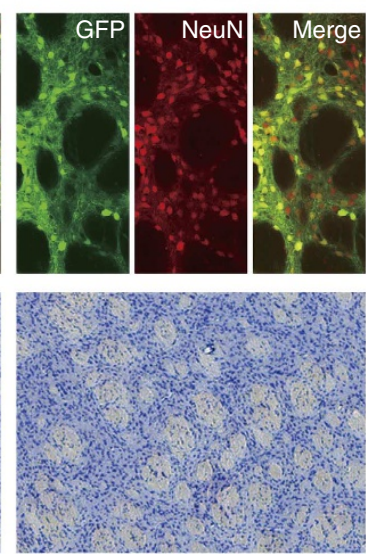

d
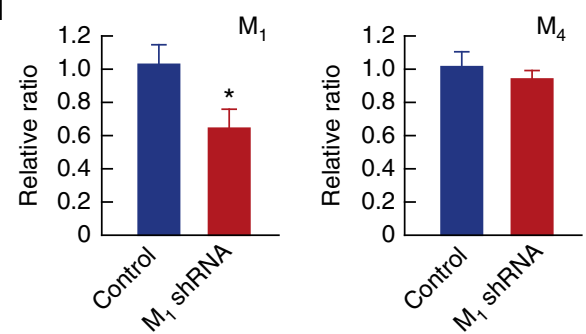

e

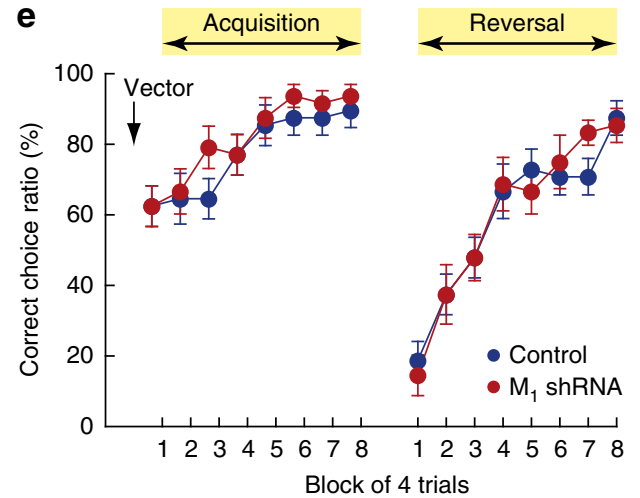

Figure 7 | Gene silencing of $\mathbf{M}_{\mathbf{1}}$ muscarinic receptor in the striatum does not change behavioural switching. Long Evans rats were given a unilateral or bilateral injection of lentivectors encoding $M_{1}$ shRNA and non-target control shRNA in the DMS and tested for histologic, biochemical and behavioural analyses. (a) Gene transduction areas visualized by GFP transgene in the rats received unilateral vector injection. Scale bar, $500 \mu$ m. (b) Transduction of DMS neurons revealed by double immunostaining for GFP and NeuN. Scale bar, $100 \mu m$. (c) Cresyl violet staining of striatal sections. Scale bar, $500 \mu \mathrm{m}$. (d) $M_{1}$ and $M_{4}$ muscarinic receptor mRNA levels measured by quantitative reverse transcription PCR analysis. Total RNA was extracted from the DMS of the rats that received unilateral injection and used for quantitative reverse transcription PCR analysis. The relative mRNA levels for the two receptors to $\beta$-actin level were normalized by the average of the values obtained from non-target control shRNA vector-injected side. $n=6$ for each group. ${ }^{\star} P<0.01$, relative to the control group (Student's $t$-test). (e) Performance of reversal learning of rats that received bilateral injection of lentivectors. $n=12$ for each group. Data are presented as mean \pm s.e.m.

we tested the influence of gene silencing of the receptor in the DMS on our behavioural paradigm. Five different shRNA sequences against rat $M_{1}$ muscarinic receptor gene $\left(M_{1}\right.$ shRNA-1 to -5) were designed for lentiviral vector-mediated expression (see Supplementary Fig. 8 for shRNA sequences). We selected $M_{1}$ shRNA-2 for the in vivo experiments based on the knockdown efficacy in cultured cells (Supplementary Fig. 10). Rats were given a unilateral injection of lentiviral vector encoding $\mathrm{M}_{1}$ shRNA-2 or a non-target control sequence, together with GFP transgene; striatal sections were used for histologic examinations. Gene transduction was localized in the DMS in both injected sides (Fig. 7a), and GFP/NeuN double staining showed the transduction of many neurons (Fig. $7 \mathrm{~b} ; 66.2 \%$ in $\mathrm{M}_{1}$ shRNA and $65.3 \%$ in the control) with no sign of tissue damage surrounding the injection sites (Fig. 7c). Quantitative reverse transcription PCR showed a significant decline in the $M_{1}$ muscarinic receptor mRNA levels in the shRNA vector-injected side relative to the control vector-injected one $(64.5 \%$ of the control value, $\left.t_{(10)}=3.265, P=0.008\right)$ with no change in the $\mathrm{M}_{4}$ receptor mRNA levels between both sides (Fig. 7d), indicating the specific knockdown of $\mathrm{M}_{1}$ receptor in the DMS.

For validation of the effect of $\mathrm{M}_{1}$ receptor knockdown on reversal learning, rats were given bilateral injection of the lentivector $\left(3 \times 10^{8}\right.$ transduction units $\left.\mathrm{ml}^{-1}\right)$ into the DMS and tested with the place discrimination task. The rats injected with the $\mathrm{M}_{1}$ shRNA vector acquired place discrimination in a fashion similar to the control group (Fig. 7e; group effect, $F_{(1,22)}=0.812$, $P=0.377$; block effect, $F_{(7,154)}=13.212, P=0.000$; interaction, $F_{(7,154)}=0.534, P=0.808$, two-way ANOVA). The performance of reversal learning was also indistinguishable between these two rat groups (Fig. 7e; group effect, $F_{(1,22)}=0.021, P=0.886$; block effect, $F_{(7,154)}=41.298, P=0.000$; interaction, $F_{(7,154)}=0.628$, $P=0.732$, two-way ANOVA). Therefore, selective knockdown of $M_{1}$ receptor in the DMS did not affect the performance of place reversal learning. Our results obtained from gene-silencing experiments suggest that cholinergic function in the DMS that suppresses behavioural flexibility is mediated predominantly via $\mathrm{M}_{4}$ receptor subtype.

\section{Discussion}

The major finding in the present study is that the selective targeting of striatal cholinergic interneurons, in particular from the DMS subregion, led to the enhancement of behavioural switching processes during reversal and extinction learning of place discrimination, normally sparing the acquisition of original learning. This finding highlighted, for the first time, the inhibitory role of striatal cholinergic interneurons in behavioural flexibility. In addition, gene-specific silencing of muscarinic receptor subtypes in the DMS by shRNA expression demonstrated that the action of $\mathrm{M}_{4}$ muscarinic receptor, but not $\mathrm{M}_{1}$ muscarinic receptor, suppresses flexible switching of behaviours. Taken together, our results suggest that the inhibitory role of striatal cholinergic interneurons in behavioural flexibility is mediated mainly through the action of $\mathrm{M}_{4}$ muscarinic receptor subtype.

Previous pharmacologic studies report that the striatal acetylcholine facilitates reversal learning of response or place discrimination ${ }^{25-28}$. A recent study using a goal-directed learning paradigm also supports the positive effects of striatal cholinergic interneurons in the reversal of action-outcome contingency ${ }^{37}$. In contrast, our present data indicate that the striatal cholinergic interneurons inhibit behavioural flexibility and that this function 
is mainly mediated through $\mathrm{M}_{4}$ muscarinic receptor in the DMS. One possible explanation for the contradictory results between our study and previous ones may lie in the drug spectrum of receptor subtypes. The conclusions of some previous studies are based on the pharmacologic efficacy of oxotremorine sesquifumarate, an $\mathrm{M}_{2} / \mathrm{M}_{4}$ muscarinic agonist with a higher affinity to presynaptic $M_{2}$ muscarinic receptor that blockades acetylcholine efflux and disrupts behavioural flexibility ${ }^{28,37}$. However, oxotremorine sesquifumarate is also known to possess affinity for $\mathrm{M}_{4}$ muscarinic receptor ${ }^{29}$. This receptor subtype is localized in the majority of striatal projection neurons ${ }^{33,34}$, and its allosteric effect reverses hyperactivity induced by psychostimulants ${ }^{35,36}$. Therefore, it may be possible that oxotremorine sesquifumarate affects the function of postsynaptic $\mathrm{M}_{4}$ receptor, which results in the impaired behavioural flexibility.

In addition, previous pharmacologic studies report a facilitative role of $M_{1}$ muscarinic receptor in reversal learning ${ }^{26,27}$. However, gene silencing of $M_{1}$ receptor described herein showed no significant impact on our behavioural paradigm. One possible explanation is that the short-term effect of $M_{1}$ receptor blockade may be masked by some compensatory mechanisms of receptor function in gene-silencing experiments. $M_{1}$ receptor signalling is known to be modulated through phosphorylation of the receptor by a variety of receptor kinases and second messenger-regulated kinases, such as G-protein-coupled receptor kinase 2 and protein kinase $\mathrm{C}^{38}$. G-protein-coupled receptor kinase 2 also regulates $\mathrm{M}_{1}$ muscarinic receptor via a phosphorylation-independent mechanism ${ }^{39}$. The possibility of whether such regulatory mechanisms of receptor function compensate for the behavioural performance remains to be examined. Another possibility may originate from the strategic difference in the behavioural paradigms between those studies. In the previous studies, rats were tested for acquisition and reversal learning on two consecutive days and the inter-trial interval was $15 \mathrm{~s}^{26,27}$, whereas in our paradigm, animals were tested for these two phases across eight consecutive days, respectively, with an intertrial interval of $20 \mathrm{~min}$. This strategic difference may generate a distinction in the memory processing requirement between the paradigms, suggesting the presence of different aspects of reversal learning that either do or do not depend on striatal $M_{1}$ muscarinic receptor.

Enhanced reversal learning in rats lacking striatal cholinergic interneurons was accompanied by improved extinction learning. Extinction is considered to be neither unlearning nor an erasure of the original learning but a kind of new learning 40 . Extinction is also known to require de novo protein synthesis ${ }^{41,42}$. Thus, the improved extinction caused by cholinergic elimination supports the role of striatal cholinergic interneurons in behavioural flexibility. In addition, analysis of error category in the reversal phase indicated a significant reduction in regressive error frequency with no change in perseverative error frequency in the rats lacking cholinergic interneurons. The data suggest that the enhanced reversal learning in these rats may involve an increased ability to learn the new strategy. However, it may be difficult to experimentally define the capacity to acquire the new associations independent of extinction processes, because the brain regions such as the orbitofrontal cortex and DMS that are implicated in reversal learning are also required for extinction learning ${ }^{43-45}$, and drug-induced impairment in reversal learning of spatial discrimination is accompanied by the inhibition of extinction ${ }^{46}$. The specific role of striatal cholinergic interneurons in the acquisition of the new associations remains to be addressed through more detailed behavioural analyses.

Behavioural flexibility promoted in rats by deleting striatal cholinergic interneurons was pharmacologically reversed by muscarinic activation in the DMS in either the acquisition or the reversal/extinction learning phase, suggesting the role of cholinergic interneurons in the two phases of learning. The cholinergic function in the acquisition phase may stabilize the contingency of rewards to the response. However, this function does not simply correspond to increases in the retention level of a previously learned strategy, since the correct choice ratio in the initial stage of reversal learning did not differ between the cell ablation and control rat groups (see Figs $1 \mathrm{~h}$ and $2 \mathrm{~d}$ ). The stabilized reward contingency may act to inhibit the switching processes of behaviours in the phase of reversal or extinction learning. Another cholinergic function in the reversal or extinction learning phase may directly suppress the processes of response switch to changed contingency.

Chemical depletion of the nigrostriatal dopamine system impairs the performance of reversal learning based on simple discrimination in rats ${ }^{47}$. Targeted disruption of dopamine $\mathrm{D}_{2}$ receptor gene also causes abnormalities in reversal learning 48 . Overexpression of the dopamine $\mathrm{D}_{2}$ receptor gene in the striatum or pharmacologic activation of $\mathrm{D}_{2} / \mathrm{D}_{3}$ receptor signalling impairs reversal learning in rodents and monkeys ${ }^{49,50}$. These data suggest that striatal dopamine transmission is engaged in the control of behavioural switching processes in response to changed contingency. In addition, striatal acetylcholine facilitates or inhibits dopamine release depending on the frequency of presynaptic activity ${ }^{51,52}$. Synchronized activation of striatal cholinergic interneurons, dependent on thalamostriatal stimulation, triggers dopamine release $\mathrm{e}^{53}$. Activation of dopamine receptors modulates the firing and synaptic plasticity of striatal cholinergic interneurons ${ }^{54,55}$. Interaction between acetylcholine and dopamine transmission in the striatum may underlie the neural mechanism that regulates behavioural flexibility.

Direct or indirect pharmacologic activation of the basal forebrain cholinergic systems, projecting to the cerebral cortex and hippocampus, improves cognitive deficits in neuropsychiatric and neurologic diseases ${ }^{56,57}$. In general, cholinergic functions have been considered to play essential roles in the facilitation of memory and learning. In contrast to the previous pharmacologic view, we found the inhibitory role of striatal cholinergic interneurons in reversal and extinction learning. The role of each cholinergic system in memory and learning appears to depend on the properties of behavioural paradigms. The pharmacologic approach that selectively blockades the activity of striatal cholinergic interneurons may help the efficacy of drugs that target the basal forebrain cholinergic systems.

\section{Methods}

Generation of $\mathbf{T g}$ rats. The rat ChAT bacterial artificial chromosome clone (CHO-520K7) was selected from the RPCI-22 129S6/SvEvTac genomic library (Children's Hospital Oakland Research Institute). The gene cassette encoding IL-2R $\alpha / \mathrm{mVenus}$ followed by the SV40 early-gene polyadenylation signal was introduced into the place of initiation codon of the rat ChAT gene with a Red/ET quick \& easy BAC modification kit (Gene Bridges). The transgene construct was linearized by Sfi digestion and purified by pulse field gel electrophoresis. The construct was microinjected into fertilized Long Evans rat eggs, which were implanted into pseudopregnant females. Tg rats were identified by southern blot hybridization or PCR with genomic DNA prepared from tail clips. All animal experiments were approved and performed in accordance with the guidelines for the care and use of laboratory animals established by the Animal Experiments Committee of Fukushima Medical University.

Viral vector production. Lentiviral vectors pseudotyped with vesicular stomatitis virus glycoprotein were generated by DNA transfection ${ }^{58}$. The GFP expression cassette under the control of CAG promoter was introduced upstream of woodchuck hepatitis virus posttranscriptional regulatory element in the transfer plasmid pCS-CA-MCS (RIKEN BioResource Center), and then the human U6 promoter flanked by an additional sequence containing BsmBI and XhoI restriction enzyme sites was inserted upstream of the CAG promoter, resulting in the plasmid termed pCS-U6-CA-GFP. Small-interference RNA sequences against rat $\mathrm{M}_{4} / \mathrm{M}_{1}$ 
muscarinic receptor gene were designed by BLOCK-iT RNAi Designer (Invitrogen), and a non-target control sequence was obtained from Sigma-Aldrich. Synthetic oligonucleotides for top and bottom sequences corresponding to shRNAs (see Supplementary Fig. 8) were annealed, phosphorylated and cloned between BsmBI and XhoI sites in the plasmid pCS-U6-CA-GFP. HEK293T cells were transfected with transfer, envelope, and packaging plasmids using lipofectamine LTX (Invitrogen). Vector particles were collected by ultracentrifugation at $100,000 \mathrm{~g}$ for $1 \mathrm{~h}$ and resuspended in PBS. HEK293T cells were seeded onto 24-well culture plates and transduced with proper concentrations of viral vectors, and the functional titre was measured by flow cytometry (FACSCalibur, Becton Dickinson).

Intracranial surgery. Rats (11-14 weeks old) were anesthetized with sodium pentobarbital ( $50 \mathrm{mg} \mathrm{kg}^{-1}$, intraperitoneal) and subjected to unilateral or bilateral intrastriatal injection of IT solution $\left(5 \mu \mathrm{g} \mathrm{ml}^{-1}\right.$ anti-Tac(Fv)-PE38 (ref. 59) in PBS containing $0.1 \%$ rat serum albumin). For targeting of cholinergic interneurons in the whole region of the dorsal striatum, solution was injected into six sites $(1.0 \mu \mathrm{l}$ per site) of the striatum through a glass micropipette, which was stereotaxically introduced by using the coordinates from an atlas of the rat brain ${ }^{60}$. For subregionspecific targeting of interneurons, solution was injected into six sites $(0.35 \mu \mathrm{l}$ per site) in the DMS or DLS. The anteroposterior, mediolateral and dorsoventral coordinates $(\mathrm{mm})$ from bregma and dura were $(1.8,2.3,-3.8),(1.8,3.0,-4.5)$, $(1.1,2.4,-3.8),(1.1,3.3,-4.5),(0.35,2.7,-4.1)$ and $(0.35,3.7,-4.2)$ for injection into the whole dorsal striatum; $(1.8,1.8,-3.3),(1.8,1.8,-3.8),(1.1,2.0$, $-3.3),(1.1,2.0,-3.8),(0.3,2.3,-3.6)$ and $(0.3,2.3,-4.2)$ for injection into the DMS; and $(1.8,3.4,-3.8),(1.8,3.4,-4.5),(1.1,4.2,-3.8),(1.1,4.2,-4.5)$, $(0.3,4.4,-3.6)$ and $(0.3,4.4,-4.1)$ for injection into the DLS. Injection was carried out at a constant flow rate of $0.5 \mu \mathrm{min}^{-1}$ with a microinfusion pump.

For infusion of muscarinic receptor drugs, rats were surgically, bilaterally implanted with 22-gauge guide cannulae (Plastics One) into the DMS using the coordinates anteroposterior 1.0, mediolateral 2.3 and dorsoventral $-2.5 \mathrm{~mm}$ from bregma and dura according to the rat brain atlas ${ }^{60}$. Solution containing OxoM $\left(8.0 \mu \mathrm{g} \mathrm{ml}^{-1}, 0.5 \mu \mathrm{l}\right.$ per site, Sigma) was infused into the DMS through 28-gauge internal cannulae (Plastic One) at a flow rate of $0.25 \mu \mathrm{lmin}^{-1}$. Drug infusion was performed $10 \mathrm{~min}$ prior to each daily trial.

Rats (9-12 weeks old) were anesthetized with sodium pentobarbital and subjected to viral vector injection. Vector solution $\left(3 \times 10^{8}\right.$ transduction units $\left.\mathrm{ml}^{-1}\right)$ was bilaterally introduced into three sites $(1.0 \mu \mathrm{l}$ per site) in the DMS through a glass microinjection capillary at a constant flow speed of $0.1 \mu \mathrm{min}^{-1}$. The anteroposterior, mediolateral and dorsoventral coordinates $(\mathrm{mm})$ from bregma and dura were $1.8 / 2.3 /-3.6,1.0 / 2.6 /-3.6$ and $0.2 / 2.8 /-3.6$.

Histology. Brain sections were incubated with primary antibodies for GFP (rabbit, 1:2,000, Life Technologies), ChAT (mouse, 1:1,000, Millipore), parvalbumin (rabbit, 1:1,000, Sigma-Aldrich), somatostatin (rabbit, 1:1,000, Dako), calretinin (rabbit, 1:1,000, Millipore), tyrosine hydroxylase (mouse, 1:300, Millipore) and vesicular glutamate transporters 1/2 (rabbit, 1:1,000, Synaptic Systems), and then with biotinylated or fluorescein isothiocyanate-conjugated secondary antibodies. The immunoreactive signals were visualized by using a Vectastain Elite ABC kit. For double immunofluorescence histochemistry, the sections were incubated with anti-GFP antibody and anti-ChAT or anti-NeuN (mouse, 1:500, Millipore) antibodies, and then with species-specific secondary antibodies conjugated to Alexa488 (Molecular probes) and Cy3 (Jackson ImmunoResearch). For cell counts, the number of ChAT-positive cells in a region of interest $(2 \times 2 \mathrm{~mm}$ for the dorsal striatum; $1 \times 1 \mathrm{~mm}$ for the DMS/DLS) was counted, and five sections from a single animal were selected for cell counts.

For in situ hybridization, sections were hybridized with an antisense RNA probe for human IL-2R $\alpha$ labelled by using in vitro transcription with digoxigenin-11UTP (Roche). The signals were visualized with anti-digoxigenin Fab fragment conjugated to alkaline phosphatase (Roche).

Quantitative reverse transcription PCR analysis. The brain was cut into $0.3-\mathrm{mm}$ slices by using a microslicer (Dosaka EM) and the DMS region was dissected out with tungsten needles under a stereomicroscope. Total RNA was extracted from the brain tissue with TRIzol reagent (Invitrogen). Reverse transcription of total RNA $(0.5 \mu \mathrm{g})$ was performed with a ReverTra Ace qPCR RT Master Mix (TOYOBO Life Science). After first-strand cDNA synthesis, quantitative PCR was performed using Power SYBR Green PCR Master Mix Reagents and a 7300 Real-Time PCR System piloted by Sequence Detection Software v1.4 (Applied Biosystems). Relative mRNA levels of $\mathrm{M}_{4}$ and $\mathrm{M}_{1}$ muscarinic receptors were calculated based on the $\Delta \Delta \mathrm{Ct}$ method by normalization using the Ct-value of rat $\beta$-actin mRNA. PCR primers used were $5^{\prime}$-ACAGCCAGGTAGACAGGCAGG- $3^{\prime}$ and $5^{\prime}$-GTGGTGACAGTTCA GACCCTCC $-3^{\prime}$ for rat $\mathrm{M}_{4}$ receptor gene, $5^{\prime}$-CCTGAGCTACCGAGCCAAGC- $3^{\prime}$ and $5^{\prime}$-CCCAACTAGGTATTGCCAGAAG- $3^{\prime}$ for rat $\mathrm{M}_{1}$ receptor gene, and $5^{\prime}$-CCGTCTTCCCCTCCATCGTG- $3^{\prime}$ and $5^{\prime}$-CCAGTTGGTGACAATGCCG TGT- $3^{\prime}$ for rat $\beta$-actin gene.

Behavioural analysis. Age-matched littermates of male rats (12-15 weeks old) were used for behavioural tests, including the place discrimination and open field activity tasks. In the place discrimination task, a black acrylic four-arm cross maze was modified to the T-shape by preventing entrance into one of the arms with a removable plastic wall (width $\times$ height, $11.8 \times 30 \mathrm{~cm}$ ). The arms in the maze were of equal size (length $\times$ width, $58 \times 11.8 \mathrm{~cm}$ ) and radiated horizontally from a central square area $(11.8 \times 11.8 \mathrm{~cm})$. The maze was positioned to face each arm to ideal north, south, east and west, respectively. One set of two opposite arms (for example, north and south) were used as choice arms, and another set of the arms (for example, east and west) were used as start arms. A recessed food cup $(3 \mathrm{~cm}$ in diameter, $1.6 \mathrm{~cm}$ in height) was placed at the end of each choice arm. The animals behaviour was monitored using an overhead CCD camera connected to a digital video cassette recorder.

The place discrimination task consisted of three phases including the familiarization, acquisition and reversal/extinction learning phase. In the familiarization phase, rats were placed in the central area of the maze and allowed to move freely and get rewards from both choice arms, which were baited with $300 \mu \mathrm{l}$ of $30 \%$ sucrose solution. In the acquisition phase, one choice arm (correct arm) of the maze was reinforced by $100 \mu \mathrm{l}$ of $30 \%$ sucrose solution, whereas another choice arm (incorrect arm) was not reinforced. The rats entered from one of the start arms in a pseudorandom order, and were required to select the correct arm. The rats completed a block of four training trials every day (the inter-trial interval of $20 \mathrm{~min}$ ) for 8 consecutive days. In the reversal phase, the positions of correct and incorrect arms were reversed. All remaining protocols were the same as those used in the acquisition phase. For extinction, neither of the choice arms was reinforced, and the trial was terminated when the rats selected either choice arm or after 10 min elapsed. The ratio of selecting the arm that had rewarded in the previous phase was measured to evaluate extinction learning. The animals included in the extinction experiment were naïve rats rather than ones that had already undergone the reversal experiment.

An open field apparatus consisted of grey plastic wall and base (width $\times$ depth $\times$ height, $70 \times 70 \times 40 \mathrm{~cm}$ ). The base was divided into 49 virtual units $(10 \times 10 \mathrm{~cm}$ square $)$ to count unit crossings. Total unit crossings and average velocity of ambulation for every 10-min session during a 60 -min test period were measured by using a video tracking software (Biobserve)

After the behavioural tests, brain sections were processed for the histologic examination to confirm the cell loss in the targeted brain areas. The rats were injected with $4 \%$ pontamine sky blue $\left(0.5 \mu \mathrm{l}, 0.25 \mu \mathrm{min}^{-1}\right)$ through the internal cannulae, and the staining was observed for the placement sites of the cannulae.

Statistical analysis. The ANOVA, post hoc Bonferroni method and Student's $t$-test were used for statistical comparisons. $P$-values $<0.05$ indicated statistical significance.

\section{References}

1. Miller, E. K. \& Cohen, J. D. An integrative theory of prefrontal cortex function. Annu. Rev. Neurosci. 24, 167-202 (2001)

2. McAlonan, K. \& Brown, V. J. Orbital prefrontal cortex mediates reversal learning and not attentional set shifting in the rat. Behav. Brain Res. 146, 97-103 (2003).

3. Raggozzino, M. E., Kim, J., Hassert, D., Minniti, N. \& Kiang, C. The contribution of the rat prelimbic-infralimbic areas to different forms of task switching. Behav. Neurosci. 117, 1054-1065 (2003).

4. Boulougouris, V., Dalley, J. W. \& Robbins, T. W. Effects of orbitofrontal, infralimbic and prelimbic cortical lesions in serial spatial reversal learning in the rat. Behav. Brain Res. 179, 219-228 (2007).

5. Clarke, H. F., Robbins, T. W. \& Roberts, A. C. Lesions of the medial striatum in monkeys produce perseverative impairments during reversal learning similar to those produced by lesions of the orbitofrontal cortex. J. Neurosci. 28, 10972-10982 (2008).

6. Castañé, A., Theobald, D. E. \& Robbins, T. W. Selective lesions of the dorsomedial striatum impair serial spatial reversal learning in rats. Behav. Brain Res. 210, 74-83 (2010)

7. Braun, S. \& Hauber, W. The dorsomedial striatum mediates flexible choice behavior in spatial tasks. Behav. Brain Res. 220, 288-293 (2011).

8. Gabbott, P. L., Warner, T. A., Jays, P. R., Salway, P. \& Busby, S. J. Prefrontal cortex in the rat: projections to subcortical autonomic, motor, and limbic centers. J. Comp. Neurol. 492, 145-177 (2005).

9. McGeorge, A. J. \& Faull, R. L. The organization of the projection from the cerebral cortex to the striatum in the rat. Neuroscience 29, 503-537 (1989).

10. Krugel, L. K., Biele, G., Mohr, P. N., Li, S.-C. \& Heekeren, H. R. Genetic variation in dopaminergic neuromodulation influences the ability to rapidly and flexibly adapt decisions. Proc. Natl Acad. Sci. USA 106, 17951-17956 (2009).

11. Stelzel, C., Basten, U., Montag, C., Reuter, M. \& Fiebach, C. J. Frontostriatal involvement in task switching depends on genetic differences in D2 receptor density. J. Neurosci. 30, 14205-14212 (2010).

12. Kehagia, A. A., Murray, G. K. \& Robbins, T. W. Learning and cognitive flexibility: frontostriatal function and monoaminergic modulation. Curr. Opin. Neurobiol. 20, 199-204 (2010). 
13. Floresco, S. B., Zhang, Y. \& Enomoto, T. Neural circuits subserving behavioral flexibility and their relevance to schizophrenia. Behav. Brain Res. 204, 396-409 (2009).

14. Goldberg, T. E. \& Weinberger, D. R. Probing prefrontal function in schizophrenia with neuropsychological paradigms. Schizophr. Bull. 14, 179-183 (1988).

15. Morice, R. Cognitive inflexibility and pre-frontal dysfunction in schizophrenia and mania. Br. J. Psychiatry 157, 50-54 (1990).

16. Smith, A. B., Taylor, E., Brammer, M., Toone, B. \& Rubia, K. Task-specific hypoactivation in prefrontal and temporoparietal brain regions during motor inhibition and task switching in medication-naïve children and adolescents with attention deficit hyperactivity disorder. Am. J. Psychiatry 163, 1044-1051 (2006).

17. Rubia, K. et al. Disorder-specific inferior prefrontal hypofunction in boys with pure attention-deficit/hyperactivity disorder compared to boys with pure conduct disorder during cognitive flexibility. Hum. Brain Mapp. 31, 1823-1833 (2010).

18. Aosaki, T. et al. Responses of tonically active neurons in the primate's striatum undergo systematic changes during behavioral sensorimotor conditioning. J. Neurosci. 14, 3969-3984 (1994).

19. Yamada, H., Matsumoto, N. \& Kimura, M. Tonically active neurons in the primate caudate nucleus and putamen differentially encode instructed motivational outcomes of action. J. Neurosci. 24, 3500-3510 (2004).

20. Apicella, P. Leading tonically active neurons of the striatum from reward detection to context recognition. Trends Neurosci. 30, 299-306 (2007).

21. Kaneko, S. et al. Synaptic integration mediated by striatal cholinergic interneurons in basal ganglia function. Science 289, 633-637 (2000).

22. Kitabatake, Y., Hikida, T., Watanabe, D., Pastan, I. \& Nakanishi, S. Impairment of reward-related learning by cholinergic cell ablation in the striatum. Proc. Natl Acad. Sci. USA 100, 7965-7970 (2003).

23. Chang, Q. \& Gold, P. E. Switching memory systems during learning: changes in patterns of brain acetylcholine release in the hippocampus and striatum in rats. J. Neurosci. 23, 3001-3005 (2003).

24. Brown, H. D., Baker, P. M. \& Ragozzino, M. E. The parafascicular thalamic nucleus concomitantly influences behavioral flexibility and dorsomedial striatal acetylcholine output in rats. J. Neurosci. 30, 14390-14398 (2010).

25. Ragozzino, M. E., Jih, J. \& Tzavos, A. Involvement of the dorsomedial striatum in behavioral flexibility: role of muscarinic cholinergic receptors. Brain Res. 953, 205-214 (2002).

26. Tzavos, A., Jih, J. \& Ragozzino, M. E. Differential effects of M1 muscarinic receptor blockade and nicotinic receptor blockade in the dorsomedial striatum on response reversal learning. Behav. Brain Res. 154, 245-253 (2004).

27. McCool, M. F., Patel, S., Talati, R. \& Ragozzino, M. E. Differential involvement of M1-type and M4-type muscarinic cholinergic receptors in the dorsomedial striatum in task switching. Neurobiol. Learn. Mem. 89, 114-124 (2008).

28. Ragozzino, M. E., Mohler, E. G., Prior, M., Palencia, C. A. \& Rozman, S. Acetylcholine activity in selective striatal regions supports behavioural flexibility. Neurobiol. Learn. Mem. 91, 13-22 (2009).

29. Bräuner-Osborne, H. \& Brann, M. R. Pharmacology of muscarinic acetylcholine receptor subtypes (m1-m5): high throughput assays in mammalian cells. Eur. J. Pharmacol. 295, 93-102 (1996).

30. Kobayashi, K. et al. Immunotoxin-mediated conditional disruption of specific neurons in transgenic mice. Proc. Natl Acad. Sci. USA 92, 1132-1136 (1995).

31. Kobayashi, K., Okada, K. \& Kai, N. in Controlled genetic manipulations (Neuromethods) Vol. 65 (eds Allexei, M.) 193-205 (Humana, 2012).

32. Fukabori, R. et al. Striatal direct pathway modulates response time in execution of visual discrimination. Eur. J. Neurosci. 35, 784-797 (2012).

33. Hersch, S. M., Gutekunst, C. A., Rees, H. D., Heilman, C. J. \& Levey, A. I. Distribution of $\mathrm{m} 1-\mathrm{m} 4$ muscarinic receptor proteins in the rat neostriatum: light and electron microscopic immunocytochemistry using subtype-specific antibodies. J. Neurosci. 14, 3351-3363 (1994).

34. Zhang, W., Yamada, M., Gomeza, J., Basile, A. S. \& Wess, J. Multiple muscarinic acetylcholine receptor subtypes modulate striatal dopamine release, as studied with M1-M5 muscarinic receptor knock-out mice. J. Neurosci. 22, 6347-6352 (2002).

35. Chan, W. Y. et al. Allosteric modulation of the muscarinic $\mathrm{M}_{4}$ receptor as an approach to treating schizophrenia. Proc. Natl Acad. Sci. USA 105, 10978-10983 (2008)

36. Brady, A. E. et al. Centrally active allosteric potentiators of the $\mathrm{M}_{4}$ muscarinic acetylcholine receptor reverse amphetamine-induced hyperlocomotor activity in rats. J. Pharmacol. Exp. Ther. 327, 941-953 (2008).

37. Bradfield, L. A., Bertran-Gonzalez, J., Chieng, B. \& Balleine, B. W. The thalamostriatal pathway and cholinergic control of goal-directed action: interlacing new with existing learning in the striatum. Neuron 79, 153-166 (2013).

38. Haga, K. et al. Phosphorylation of human $\mathrm{ml}$ muscarinic acetylcholine receptors by $\mathrm{G}$ protein-coupled receptor kinase 2 and protein kinase C. J. Biol. Chem. 271, 2776-2782 (1996).
39. Willets, J. M., Nash, M. S., Challiss, R. A. J. \& Nahorski, S. R. Imaging of muscarinic acetylcholine receptor signaling in hippocampal neurons: evidence for phosphorylation-dependent and -independent regulation by G-protein-coupled receptor kinases. J. Neurosci. 24, 4157-4162 (2004).

40. Bouton, M. E. Context, ambiguity, and unlearning: sources of relapse after behavioural extinction. Biol. Psychiatry 52, 976-986 (2002).

41. Berman, D. E. \& Dudai, Y. Memory extinction, learning anew, and learning the new: dissociations in the molecular machinery of learning in cortex. Science 291, 2417-2419 (2001).

42. Vianna, M. R., Szapiro, G., McGaugh, J. L., Medina, J. H. \& Izquierdo, I. Retrieval of memory for fear-motivated training initiates extinction requiring protein synthesis in the rat hippocampus. Proc. Natl Acad. Sci. USA 98, 12251-12254 (2001)

43. Izquierdo, A. \& Murray, E. A. Combined unilateral lesions of the amygdala and orbital prefrontal cortex impair affective processing in rhesus monkeys J. Neurophysiol. 91, 2023-2039 (2004).

44. Izquierdo, A. \& Murray, E. A. Opposing effects of the amygdala and orbital prefrontal cortex lesions on the extinction of instrumental responding in macaque monkeys. Eur. J. Neurosci. 22, 2341-2346 (2005).

45. Corbit, L. H. \& Janak, P. H. Inactivation of the lateral but not medial dorsal striatum eliminates the excitatory impact of Pavlovian stimuli on instrumental responding. J. Neurosci. 27, 13977-13981 (2007).

46. Christoffersen, G. R. J., Kemp, A. \& Örlygsdottir, G. Piracetam inhibits Pavlovian extinction and reversal learning in a spatial task for rats. Neuropharmacology 37, 815-825 (1998).

47. O'Neill, M. \& Brown, V. J. The effect of striatal dopamine depletion and the adenosine $\mathrm{A}_{2 \mathrm{~A}}$ antagonist KW-6002 on reversal learning in rats. Neurobiol. Learn. Mem. 88, 75-81 (2007).

48. Kruzich, P. J. \& Grandy, D. K. Dopamine $\mathrm{D}_{2}$ receptors mediate two-odor discrimination and reversal learning in C57BL/6 mice. BMC Neurosci. 5, 12 (2004).

49. Kellendonk, C. et al. Transient and selective overexpression of dopamine D2 receptors in the striatum causes persistent abnormalities in prefrontal cortex functioning. Neuron. 49, 603-615 (2006).

50. Lee, B., Groman, S., London, E. D. \& Jentsch, J. D. Dopamine $\mathrm{D}_{2} / \mathrm{D}_{3}$ receptors play a specific role in the reversal of a learned visual discrimination in monkeys Neuropsychopharmacology 32, 2125-2134 (2007).

51. Zhang, H. \& Sulzer, D. Frequency-dependent modulation of dopamine release by nicotine. Nat. Neurosci. 7, 581-582 (2004).

52. Cragg, S. J. Meaningful silences: how dopamine listens to the ACh pause. Trends Neurosci. 29, 125-131 (2006).

53. Threlfell, S. et al. Striatal dopamine release is triggered by synchronized activity in cholinergic interneurons. Neuron. 75, 58-64 (2012).

54. Yan, Z. \& Surmeier, D. J. $\mathrm{D}_{5}$ dopamine receptors enhance $\mathrm{Zn}^{2+}$-sensitive $\mathrm{GABA}_{\mathrm{A}}$ currents in striatal cholinergic interneurons through a PKA/PP1 cascade. Neuron. 19, 1115-1126 (1997).

55. Suzuki, T., Miura, M., Nishimura, K. \& Aosaki, T. Dopamine-dependent synaptic plasticity in the striatal cholinergic interneurons. J. Neurosci. 21, 6492-6501 (2001).

56. Deiana, S., Platt, B. \& Riedel, G. The cholinergic system and spatial learning. Behav. Brain Res. 221, 389-411 (2011).

57. Bubser, M., Byun, N., Wood, M. R. \& Jones, C. K. Muscarinic receptor pharmacology and circuitry for the modulation of cognition. Handb. Exp. Pharmacol. 121-166 (2012).

58. Miyoshi, H., Blömer, U., Takahashi, M., Gage, F. H. \& Verma, I. M. Development of a self-inactivating lentivirus vector. J. Virol. 72, 8150-8157 (1998).

59. Kreitman, R. J., Bailon, P., Chaudhary, V. K., FitzGerald, D. J. \& Pastan, I. Recombinant immunotoxins containing anti- $\mathrm{Tac}(\mathrm{Fv})$ and derivatives of Pseudomonas exotoxin produce complete regression in mice of an interleukin-2 receptor-bearing human carcinoma. Blood 83, 426-434 (1994).

60. Paxinos, G. \& Watson, C. The Rat Brain in Stereotaxic Coordinates, 6th edn (Academic Press, 2007)

\section{Acknowledgements}

This work was supported by Grants-in-Aid for Scientific Research (B) and Scientific Research on Innovative Areas (Comprehensive Brain Science Network) from the Ministry of Education, Culture, Sports, Science and Technology of Japan; and Core Research for Evolutional Science and Technology of Japan Science and Technology Agency. We thank Dr. I. Pastan for providing anti-Tac(Fv)-PE38. We are grateful to M. Kikuchi, N. Sato, K. Sato, T. Kobayashi and S. Matsushita for their technical support in biochemical, histological and animal experiments.

\section{Author contributions}

K.O. and K.N. are credited as equal authors in this work. K.O., K.N. and K.K. conceived the study, designed the experiments and directed the project. K.O., A.S., M.U. and K.K. designed the transgene construct and generated Tg rats. N.M. designed the shRNA experiments and produced lentiviral vectors. K.O., K.N., R.F. and N.K. performed intracranial injection and histologic examination. K.O., K.N., Y.T., and S.S. designed the 
place discrimination strategy and performed the behavioural analyses. K.O., K.N. and K.K. wrote the paper. All authors discussed the results and implications and commented on the manuscript at all stages.

\section{Additional information}

Supplementary Information accompanies this paper at http://www.nature.com/ naturecommunications
Competing financial interests: The authors declare no competing financial interests.

Reprints and permission information is available online at http://npg.nature.com/ reprintsandpermissions/

How to cite this article: Okada, K. et al. Enhanced flexibility of place discrimination learning by targeting striatal cholinergic interneurons. Nat. Commun. 5:3778 doi: $10.1038 /$ ncomms4778 (2014). 